\title{
Fidelity Decay in Chaotical and Random Systems
}

\author{
H. KOHLER* \\ Instituto de Ciencia de Materiales de Madrid, CSIC, Sor Juana de la Cruz 3 \\ Cantoblanco, 28049 Madrid, Spain
}

\begin{abstract}
Fidelity is the overlap of wave functions with the same initial state propagated in time by slightly different Hamiltonians. Its behavior depends crucially on the choice of the initial wave function state. We review two cases: first, the initial state is random. In this case a simple analytic relation with parametric spectral correlations can be established. The latter quantity is completely determined by the spectral data and can therefore be measured, without knowledge about the wave function. Second, the initial state is an eigenstate of the unperturbed system. In this case fidelity is identical to the survival probability. We find unexpected features like revival and non-ergodicity. In this case fluctuations around the mean are large and the full fidelity distribution becomes a non-trivial function. The full fidelity distribution can be calculated in the long time limit and for small perturbations.
\end{abstract}

PACS: 05.30.-d, 03.65.Yz, 05.45.Mt, 73.23.-b

\section{Introduction}

In classically chaotic systems nearby trajectories diverge with a rate given by the highest (or a mean) Lyapunov exponent.

There exists no obvious quantum mechanical counterpart to the classical Lyapunov exponent. However due to Ehrenfest's theorem expectation values with respect to "almost" classical states, like Gaussian wave packets, behave for a certain time according to the (in general non-linear) classical equations of motion. The wave function, respectively its Wigner function, is expected to behave similar to a classical phase space distribution until the particle starts to feel that it is actually a wave. Up to this time a Lyapunov exponent should show up even for a genuine quantum system. But is there an equivalent to the Lyapunov exponent in chaotic quantum systems deep in the quantum regime, i.e. for times well beyond the Ehrenfest time?

Even in the most perfectly prepared experiment time evolution is never completely deterministic. There exist always small fluctuations around the known Hamiltonian $H_{0}$, which includes all well controlled parts of the dynamics. One might conjecture that in quantum mechanics the main source of randomness in the outcome of an experiment is the incomplete knowledge on the time evolution rather than the uncertainty in the initial conditions like in classical mechanics. Peres was one of the first who addressed the above question [1]. He looked at the susceptibility of a system under small variations of $H_{0}$.

\footnotetext{
* corresponding author; e-mail: hkohler@icmm.csic.es
}

He defined fidelity $F(t)$, often called the Loschmidt $e c h o$, as the modulus squared of the overlap integral between an initial wave function, which is propagated in time by a Hamiltonian $H_{0}$ with the same initial wave function, which is propagated by a slightly perturbed Hamiltonian $H=H_{0}+V$ :

$$
f(t)=\left\langle\psi\left|\mathrm{e}^{\mathrm{i} H t} \mathrm{e}^{-\mathrm{i} H_{0} t}\right| \psi\right\rangle, \quad F(t)=|f(t)|^{2},
$$

where $\hbar=1$. The quantity $f(t)$ is called fidelity amplitude. Fidelity and fidelity amplitude will in general decay as function of time and of the mean perturbation strength. Peres argued that for a classically chaotic quantum system this decay will be much faster than for classically integrable systems. It turned out that the behavior of fidelity is much more complex than anticipated in his early work. It depends crucially on the many parameters, which enter in the definition. Nowadays fidelity has become object of intensive study in many different fields $[2,3]$.

Historically, most people working in the characterization of quantum chaotic systems focused on properties of their spectra. For one reason, because in nuclear physics, the parent of quantum chaos, spectral data abound and yield an ideal environment for statistical spectral analysis. On the other hand, time resolved data are rare. Also modern billiard experiments focus mostly on the measurement of the spectrum, respectively of scattering cross-sections [4].

Yet there are interesting questions like the above mentioned question for the stability of a quantum system against perturbations, where observables defined in the time domain are more appropriate than spectral observables. To see that for this specific question Peres' fidelity is a more sensitive quantity it suffices to consider an ex- 
treme case: the perturbation is isospectral, i.e. it does not change the spectrum of the unperturbed Hamiltonian at all. Any spectral quantity will remain unaffected but fidelity will in general decay.

\section{A random matrix model of fidelity}

Fidelity is a rather general quantity with a wide range of different behavior depending on its four parameters. Let us briefly review them:

1. The unperturbed Hamiltonian can be any type of system, ranging from nuclear physics over electronic systems in condensed matter physics and mesoscopics to typical Hamiltonians of quantum information devices. Stability of wave dynamics is most important as well for classical telecommunication or for certain questions in acoustics [5-7]. Following the original intentions of Peres we will focus mainly on chaotic quantum systems and disordered systems. More precisely we focus on systems whose universal spectral properties are well described by random matrices, such that $H_{0}$ can be chosen from one of the classical Gaussian random matrix (RMT) ensembles, the Gaussian unitary ensemble (GUE, $\beta=2$ ), the Gaussian orthogonal ensemble (GOE, $\beta=1$ ) or the Gaussian symplectic ensemble (GSE, $\beta=4$ ). The latter two have to be chosen for systems with even spin (GOE) or with odd spin (GSE) and with conserved time reversal invariance (TRI). The three ensembles are characterised by their second moments

$$
\overline{\left(H_{0}\right)_{i j}\left(H_{0}\right)_{k l}}=v^{2}\left[\delta_{i l} \delta_{k j}-\left(1-\frac{2}{\beta}\right) \delta_{i k} \delta_{j l}\right] .
$$

The variance $v$ sets the scale. It has the dimension of an energy in quantum mechanics and of a frequency in classical wave mechanics. Usually $v=1$. The RMT approach is well suited to provide a general benchmark for the fidelity decay with a minimal knowledge about $H_{0}$. More specific behaviors of fidelity which depend on non-universal properties of individual systems will not be captured. In particular, the classical Lyapunov exponent is a system specific quantity, which cannot arise from an invariant RMT model.

2. Perturbation. The stability of the dynamics of $H_{0}$ is probed by the perturbation. The latter is therefore not an unwanted feature but rather part of a measurement process. A global perturbation, as for instance a change in temperature in the fidelity experiment by Lobkis and Weaver [6], affects the entire Hilbert space and all energy levels in the same way. On the other hand, local perturbations only act a smaller subspace. For instance in Refs. $[8,9]$ the effect of local boundary-deformations of a chaotic billiard on fidelity was investigated.
In a random matrix approach a most general global perturbation is modeled by a second random matrix $V$. Fidelity amplitude is given by the ensemble average of the following RMT model:

$$
f(t)=\left\langle\psi\left|\mathrm{e}^{\mathrm{i} H t} \mathrm{e}^{-\mathrm{i} H_{0} t}\right| \psi\right\rangle,
$$

where the forward propagating Hamiltonian $H_{0}$ is taken from a Gaussian RMT ensemble and the backward propagating Hamiltonian is given by

$$
H=H_{0}+V \text {. }
$$

In the simplest case $V$ is chosen from the same ensemble as $H_{0}$. The model was first formulated in an important paper by Gorin et al. [10]. The mean strength of the perturbation is given by the variance of a matrix element of $V$ as

$$
\overline{V_{i j} V_{k l}}=\lambda^{2} D^{2} \overline{\left(H_{0}\right)_{i j}\left(H_{0}\right)_{k l}},
$$

where $D$ is the mean level spacing of the unperturbed Hamiltonian. For Gaussian random matrix ensembles in the limit of large matrix dimension the spectrum has compact support. The density of states is given by Wigner's semicircle law. In the center of the semicircle it is $\sqrt{2 N} /(\pi v)$ and thus the mean level spacing is $D=(v \pi) / \sqrt{2 N}$. This means that a typical matrix element of $V$ is small as $\mathcal{O}\left(N^{-1 / 2}\right)$. As a consequence in the large $N$ -limit the perturbation does not change at all the density of states of the original Hamiltonian. The perturbation is weak if a typical matrix element of $V$ is much smaller than the mean level spacing, i.e. if the dimensionless coupling strength $\lambda$ is much smaller than one $\lambda \ll 1$. The crossover to strong coupling takes place at $\lambda \simeq(2 \pi)^{-1}$, such that $\lambda=1$ is already a rather strong perturbation.

3. Time. The characteristic time scale of RMT models is given by the inverse of the mean level spacing $D$. It is the time needed to resolve individual levels of the system. This time is called the Heisenberg time. It is given by $t_{\mathrm{H}}=2 \pi \hbar / D$ in quantum mechanics and by $t_{\mathrm{H}}=1 / D$ in classical wave mechanics, where $D$ has the dimension of a frequency. By the universality hypothesis spectral properties become universal on the scale of the mean level spacing. Likewise time dependence becomes universal, i.e. does not depend on any other details of the system, on the scale of the Heisenberg time. In many systems the Heisenberg time is the largest available time scale. In RMT it is the only time scale. This means that other characteristic times, like for instance the Ehrenfest time are zero by default.

One should emphasize that Heisenberg time albeit being large is never infinite. Typical values are about $10 \mathrm{~ns}$ in microwave billiards [11] and about $80 \mathrm{~ms}$ in acoustical experiments [12]. In real time measurements of fidelity non-universal fluctuations 
on smaller time scales are expected.

4. The initial state. The behavior of fidelity depends crucially on the initial state chosen in Eq. (3). Here at least three choices are interesting. (I) A random initial state. This is in the spirit of the maximal entropy approach of RMT. The objective is to obtain universal results with a minimal knowledge on the system. Thus it is natural to assume minimal knowledge on the initial state $|\psi\rangle$ as well and to choose it as an incoherent superposition of an arbitrary set of orthogonal states, which span the full Hilbert space. (II) A second canonical choice is the initial state $|\psi\rangle$ as an eigenstate of the unperturbed Hamiltonian. In this case the forward evolution of $|\psi\rangle$ is trivial and fidelity $F(t)$ is identical with survival probability, i.e. the probability of finding the system in its initial state after time $t$. (III) A semiclassical initial state, like a Gaussian wave packet, deserves to be mentioned as well. For times smaller than the Ehrenfest time the wave packet behaves approximately classically and for chaotic dynamics fidelity decay is indeed governed by the Lyapunov exponents (the Lyapunov regime) [13]. The Lyapunov regime is non-universal and cannot be grasped by an RMT model. Semiclassical initial states will not be discussed in the following.

\section{Fidelity decay of a random initial state}

We first consider the situation that the wave function is initially in a random state

$$
|\psi\rangle=\sum_{n=1}^{N} \mathrm{e}^{\mathrm{i} \phi_{n}}|n\rangle .
$$

Here $\phi_{n}$ is a random phase, which is uniformly distributed over the unit circle and $|n\rangle$ is an eigenstate of the unperturbed Hamiltonian. In a billiard experiment this initial state describes the situation that one starts the observation of the wave after a time when the initially injected signal has already widely spread over the cavity and has essentially lost memory of its initial shape. The theoretical results obtained in this limit and presented in the following agree well with the fidelity measurements performed in billiards and in elastomechanical probes $[6,11]$.

The average over the random phase maps the expression for the fidelity amplitude into the form

$$
f(t)=\frac{1}{N} \operatorname{Tr} \mathrm{e}^{\mathrm{i} H t} \mathrm{e}^{-\mathrm{i} H_{0} t},
$$

which looks due to the trace almost invariant, i.e. basis independent. But it is not. This is seen, when we plug in two complete sets of eigenfunctions. Fidelity amplitude can be written as

$$
f(t)=\frac{1}{N} \sum_{n, \mu}\left|U_{n \mu}\right|^{2} \exp \left(\mathrm{i}\left(E_{\mu}-E_{n}\right) t\right),
$$

where $U$ is the unitary matrix, which has as columns the
$N$ eigenvectors of $H$ to eigenvalues $E_{\mu}, 1 \leq \mu \leq N$ expressed in the eigenbasis of $H_{0}$. The latter has eigenvalues $E_{n}, 1 \leq n \leq N$. Here and in the following Greek indices denote eigenvalues of the full Hamiltonian $H$ and Latin indices denote eigenvalues of the unperturbed Hamiltonian $H_{0}$. The occurrence of the unitary matrix $U$ indicates that fidelity contains information on the eigenfunctions.

In the last decade of the past century parametric energy correlations of disordered mesoscopic systems and of random matrices were investigated in great detail [14-19]. They quantify the response of the spectral correlations against a perturbation. They were studied and calculated for a variety of RMT ensembles. A parametric spectral form factor can be defined for parametric energy correlations by the Fourier transformation in the same way as for pure, non-parametric RMT ensembles. It is called cross-form factor in [20]. Formally it is defined as the ensemble average of

$$
\tilde{K}(t)=\frac{1}{N} \sum_{n, \mu} \exp \left(\mathrm{i}\left(E_{\mu}-E_{n}\right) t\right) .
$$

Although it is obviously a purely spectral quantity and contains no information about the eigenfunctions, the formal similarity to fidelity amplitude (8) is striking. The only difference is the additional factor $\left|U_{n \mu}\right|^{2}$ in the definition of fidelity amplitude.

The ensemble averages of both $\overline{f(t)}$ and $\overline{\tilde{K}(t)}$ were calculated for the case that $H_{0}$ and $V$ fall into the same symmetry class $[14,16,20,21]$. We do not state the rather complicated expressions here, but refer the reader to a compilation of the pertinent formulae in Ref. [20]. In Fig. 1 fidelity amplitude and cross-form factor are plotted for different values of the dimensionless perturbation strength $\lambda$. For small perturbation $(\lambda \ll 1)$ the cross-form factor acquires the familiar form of the pure ensembles with the characteristic logarithmic divergence at the Heisenberg time in the case of the symplectic ensemble. For strong perturbation $(\lambda=1)$ the curves of $\overline{f(t)}$ and of $\overline{\tilde{K}(t)}$ look alike. Both have characteristic peaks at the Heisenberg time, respectively at twice the Heisenberg time in case of the GSE.

A comparison of the exact analytic results for both quantities yields the remarkably simple relation

$$
\frac{\partial}{\partial\left(\lambda^{2}\right)} \overline{\tilde{K}(t)}=-\frac{4 \pi^{2} t^{2}}{\beta} \overline{f(t)} .
$$

It is surprising, since it relates a quantity, which contains information about the eigenfunctions, to a purely spectral quantity and offers an excellent opportunity to measure fidelity amplitude through an analysis of the spectral data.

The above relation can be generalized to the case, when the perturbation breaks the symmetry of the original Hamiltonian $H_{0}[17,22,23]$. Following the outline of Ref. [23] we write the full Hamiltonian as 


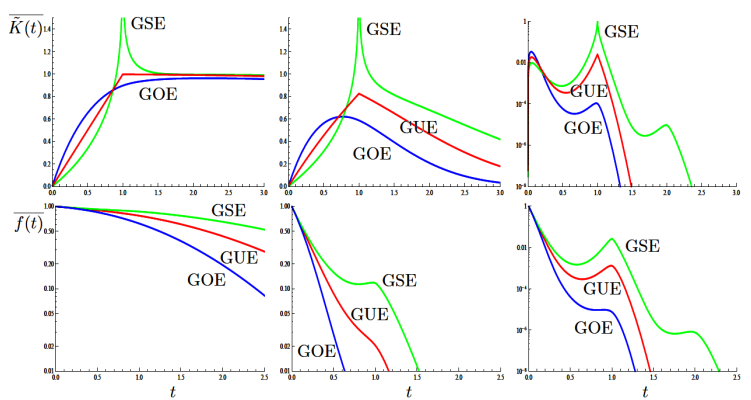

Fig. 1. Upper row: cross-form factor for three values of perturbation strength $\lambda=0.01$ (left), $\lambda=0.5$ (middle) and $\lambda=1$ (right) for GSE, GUE and GOE (from top to bottom). The last plot is on a logarithmic scale. Lower row: fidelity amplitude for the perturbation strengths $\lambda=0.1$ (left), $\lambda=0.1$ (middle) and $\lambda=1$ (right) for the GSE (green), GUE (red) and for the GOE (blue) (from top to bottom). The two last plots are on a logarithmic scale.
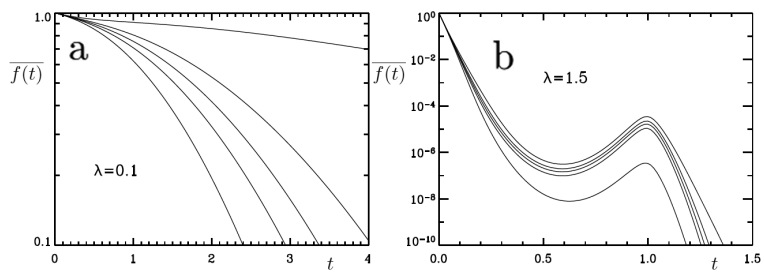

Fig. 2. Fidelity amplitude for a TRI breaking perturbation for fixed mean perturbation strength $\lambda=$ $\sqrt{\lambda_{\|}^{2}+\lambda_{\perp}^{2}}$. In part (a) fidelity amplitude is plotted for $\lambda=0.1$ and for the five ratios $\lambda_{\|} / \lambda_{\perp}=$ $\infty, \sqrt{2}, 1,1 / \sqrt{2}, 0$ (from top to bottom). In part (b) fidelity amplitude is plotted for $\lambda=1.5$ and for the same ratios from top to bottom. Pictures taken from Ref. [23].

$$
H=H_{0}+V_{\|}+V_{\perp} .
$$

The orthogonality relations $\operatorname{Tr} H_{0} V_{\perp}=0, \operatorname{Tr} V_{\|} V_{\perp}=0$ and $\operatorname{Tr} H_{0} V_{\|} \neq 0$ hold. The perturbation is thus split into a parallel subspace and into an orthogonal subspace. The two perturbations are allowed to have different coupling strengths $\lambda_{\|}$and $\lambda_{\perp}$, defined according to Eq. (5). Then relation (10) can be generalised to

$$
\frac{\partial}{\partial\left(\lambda_{\|}^{2}\right)} \overline{\tilde{K}(t)} \propto-t^{2} \overline{f(t)},
$$

i.e. only the parallel perturbation enters in the relation. In Fig. 2 fidelity amplitude is plotted for a perturbation which breaks time reversal symmetry of a system with integer spin. In this case $V_{\|}=V_{\|}^{*}$ and $V_{\perp}=-V_{\perp}^{*}$. A mean perturbation strength can be defined by $\lambda=\sqrt{\lambda_{\|}^{2}+\lambda_{\perp}^{2}}$. On the left fidelity amplitude is plotted for $\lambda=0.1$ and on the right for $\lambda=1.5$ and for five ratios $\lambda_{\|} / \lambda_{\perp}$. The decay of the fidelity amplitude increases with increasing ratio $\lambda_{\|} / \lambda_{\perp}$. Whereas for strong perturbation $(\lambda=1.5)$ the dependence on this ratio is of minor importance a drastic effect is seen for small perturbation $\lambda=0.1$. Fidelity amplitude decays much slower for $\lambda_{\|}=0$ than for finite $\lambda_{\|}$. This effect was predicted by Prosen and Znidarič in [24] to occur whenever a perturbation has zero diagonal matrix elements in the eigenbasis of $H_{0}$. There it was named "fidelity freeze". In view of the above results, we propose the following condition for a fidelity freeze:

$$
\operatorname{Tr} H_{0} V=0 .
$$

It is slightly more general and comprises the condition of Prosen and Znidarič as a special case. All states of the Hilbert space equally contribute. This is characteristic for the random initial state (6). For other initial states condition (13) will in general not suffice to yield a fidelity freeze.

\section{Fidelity of an eigenstate of the unperturbed system}

We now consider the random matrix model (3) for the case that the system is initially prepared in an eigenstate of the unperturbed system. We label this initial state $|s\rangle$ and call its eigenvalue $E_{s}$. The general expression (1) for the fidelity amplitude, respectively for fidelity, reduce in this case to

$$
f(t)=\mathrm{e}^{-\mathrm{i} E_{s} t}\left\langle s\left|\mathrm{e}^{\mathrm{i} H t}\right| s\right\rangle,
$$

and

$$
F(t)=\left|\left\langle s\left|\mathrm{e}^{\mathrm{i} H t}\right| s\right\rangle\right|^{2} .
$$

The second expression is nothing but the probability of finding the system still in state $|s\rangle$ after being propagated during a time interval $t$ by the Hamiltonian $H$. In this case fidelity is therefore called "survival probability" or - more precisely - probability of survival and return. In the following we stick with the notion "fidelity".

The matrices $H_{0}$ and $V$ are chosen either from a GOE from a GUE or Poissonian, the GSE case will be discussed elsewhere. The unperturbed Hamiltonian can be split into a system part and into a background part as

$$
H_{0}=E_{\mathrm{s}}|s\rangle\langle s|+H_{\mathrm{b}} .
$$

Likewise the perturbation $V$ has two effects. On the one hand, it perturbs the system and the background independently, on the other hand, it couples the state $|s\rangle$ to the background. The background Hamiltonian is completely characterized by its symmetry class and by its mean density of states. Since in the large $N$ limit the mean level spacing of $H_{\mathrm{b}}$ remains unaffected by $V$, the effect of the perturbation onto the background Hamiltonian should be negligible at least as long as $V$ shares the symmetry of $H_{0}$.

If $V$ breaks the symmetry of $H_{0}$, switching on the perturbation will induce a transition from GOE $\rightarrow$ GUE in the statistics of the background Hamiltonian. This effect is a priori not negligible, however our exact and numerical results confirm that the statistics of the background is of minor importance for the fidelity decay of the prepared state. 
We therefore set the part of the perturbation which perturbs solely $H_{\mathrm{b}}$ to zero right from the beginning. Likewise the perturbation of $E_{\mathrm{s}}$ is set to zero. Thus $H$ can be written conveniently in block form

$$
H=\left(\begin{array}{c|c}
E_{\mathrm{s}} & \boldsymbol{V}^{\dagger} \\
\hline \boldsymbol{V} & H_{\mathrm{b}}
\end{array}\right),
$$

where the lower right block is $(N-1) \times(N-1)$ and the upper left block is one times one. The perturbation is not full rank but rank one.

Although a rigorous proof is yet missing, by the arguments outlined above it seems plausible that the Hamiltonian (17), often called the Doorway model [25], with rank one perturbation and the full rank model (4) yield the same result for the fidelity of the initial state $|s\rangle$. One has to distinguish between a complex perturbation $V_{i} \in \mathbb{C}$ and a real perturbation $V_{i} \in \mathbb{R}$.

In a straightforward calculation [26] the mean fidelity amplitude is found to decay exponentially for any choice of $H_{\mathrm{b}}$ and of $V$ :

$$
\overline{f(t)}=\mathrm{e}^{\mathrm{i}\left(E_{s}+\mathrm{i} \Gamma / 2\right) t},
$$

where $\Gamma=2 \pi \lambda^{2} D$ is the spreading width of the local density of states,

$$
\overline{\rho(E)}=\frac{1}{\pi} \frac{\Gamma / 2}{\left(E-E_{\mathrm{s}}\right)^{2}+(\Gamma / 2)^{2}},
$$

which is as expected of the Breit-Wigner form. Calculating from this expression the fidelity in the DrudeBoltzmann approximation yields immediately Fermi's golden rule

$$
\overline{F(t)}=\overline{|f(t)|^{2}} \approx|\overline{f(t)}|^{2}=\exp (-\Gamma t) .
$$

The approximation made in Eq. (20) is similar to the approximation made in the derivation of Drude's law of classical conductivity. Fermi's golden rule predicts an exponential decay of fidelity for all times and all perturbation strength.

In order to see when corrections to this rather robust result become important, we look at the definition of fidelity

$$
F(t)=\sum_{\nu, \mu}|\langle s \mid \mu\rangle|^{2}|\langle s \mid \nu\rangle|^{2} \mathrm{e}^{\mathrm{i}\left(E_{\nu}-E_{\mu}\right) t} .
$$

In the long time limit and after averaging only the terms $\mu=\nu$ survive in the sum. We get

$$
\overline{F(\infty)}=\sum_{\nu} \overline{|\langle s \mid \nu\rangle|^{4}}
$$

The right hand side is the average inverse participation ratio $(I P R)$ of the initial state $|s\rangle$ in the basis of the eigenstates of $H$. It is a measure for the minimal distance of a vector to a basis vector of a given complete orthogonal system. For a large fragmentation of $|s\rangle$ many scalar products in the normalization $\sum_{\nu}|\langle s \mid \nu\rangle|^{2}=1$ contribute with a share of order $1 / N$ such that the IPR is of order $1 / N$ and becomes zero in the large $N$ limit. If the fragmentation of the special state $|s\rangle$ is small, a few scalar products contribute substantially to the normalization with a share of order one. These scalar products give rise to a non-zero IPR even in the large $N$ limit. An estimate for the mean IPR is obtained by

$$
\overline{I P R}=D \int \mathrm{d} E \overline{\rho(E)^{2}} \simeq \frac{D}{\pi \Gamma},
$$

where in the second equality again a Drude-Boltzmann approximation was employed. Thus IPR is non-zero, when the spreading width has the same order of magnitude as the mean level spacing $D$. The width of the Breit-Wigner curve comprises only a few neighboring energy levels of the special state $|s\rangle$. Corrections to Fermi's golden rule are therefore expected to become important when the spreading width of the state and the mean level spacing are of the same order of magnitude.

For complex entries of the vector $\boldsymbol{V}$ the ensemble average $\overline{F(t)}$ could be performed exactly in the large $N$-limit for the choices of the background Hamiltonian mentioned above, $H_{\mathrm{b}} \in$ Poisson, $H_{\mathrm{b}} \in$ GUE, $H_{\mathrm{b}} \in$ GOE. We do not state the lengthy formulae here, but refer the reader to Ref. [26].

The cases of a Poissonian background and of a GUE background are plotted in Fig. 3. We see two prominent features. First, the exact calculation confirms our qualitative arguments and shows that fidelity saturates to a finite value in the long time limit. The numerical value of this saturation value is underestimated in the approximation (23) by a factor four for a Poissonian background and by a factor two for a GUE background. Second, a revival of fidelity is observed for not too weak perturbations for a Poissonian background as well as for a GUE background.

The exact results (full lines) are plotted versus two approximate results. One approximation is Fermi's golden rule (dotted lines). It agrees well with the exact results for times up to order $\Gamma^{-1}$.

The second approximation (dashed lines) is due to Ref. [27]. There the following estimate for fidelity was found:

$$
\overline{F(t)} \simeq \mathrm{e}^{-\Gamma t}+\frac{D}{\pi \Gamma}-F_{\text {corr }}(t) .
$$

It is the sum of a Fermi golden rule contribution a constant contribution and of a correction term $F_{\text {corr }}(t)$ due to level-correlations of the background Hamiltonian. Its precise form is given in Eqs. (41) and (43) of Ref. [27]. There it was argued that this correction term is responsible for the fidelity revival seen in Fig. 3 for intermediate and strong perturbations. It occurs approximately at the same time as the exact result starts to deviate from Fermi's golden rule.

A comparison of the two plots in Fig. 3 shows that a fidelity revival occurs for a Poissonian background as well as for a GUE background. This is in contradiction to the explanation by Eq. (24), since in a Poissonian background level correlations are absent and the correction term $F_{\text {corr }}(t)$ is zero. The mechanism is clearly different to the revival observed in Figs. 1 and 2 for a random ini- 


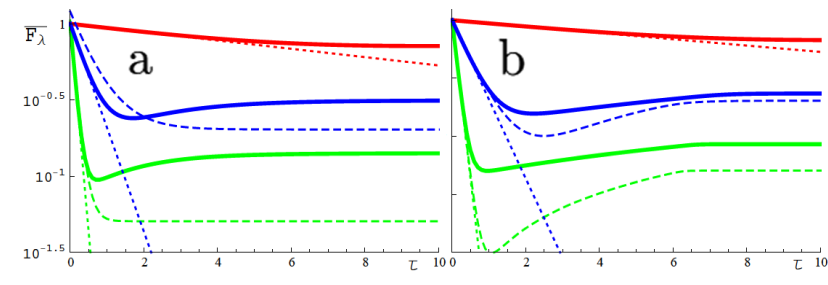

Fig. 3. Fidelity of the special state $|0\rangle$ for a regular background (b) and for a GUE background (a) for three different values of the coupling strength (from top to bottom) $\lambda=0.1$ (red), $\lambda=0.5$ (blue) and $\lambda=1$ (green). The full lines describe the exact results of Ref. [26]. The straight dotted lines are Fermi's golden rule. The dashed lines are plots of the approximate results of equations (41) and (43) of Ref. [27]. Pictures are taken from Ref. [26].

tial state. The latter occur at multiples of the Heisenberg time and are clearly a signature of spectral rigidity [28]. The revival time observed in Fig. 3 increases with $\Gamma^{-1}$.

For a GOE background the curves look quite similar to the ones for the GUE. Plots of all cases can be found in Ref. [26]. As a rule of thumb, whether the coupling matrix elements $V_{i} \in \mathbb{R}$ or $V_{i} \in \mathbb{C}$ is more important than the background complexity.

Revival and long time saturation of fidelity as predicted here has been observed experimentally in a fidelity measurement of an atom optics realization of the quantum kicked rotor [29]. Long time saturation of fidelity was predicted as well in [30] and much earlier in [31] for quantum dots in mesoscopic systems.

\section{Full fidelity statistics}

The fact that mean fidelity saturates at a finite value for $t \rightarrow \infty$ raises the question whether sample to sample fluctuations are important. To this end the full distribution function of fidelity has to be studied. It is formally defined as

$$
P_{\mathrm{F}}(c, t)=\overline{\delta(c-F(t))} \text {. }
$$

A Monte Carlo analysis reveals that indeed the initial delta-peak at $c=1$ broadens as time goes by and finally saturates in a non-trivial stable distribution for $t \rightarrow \infty$. This is shown in Fig. 4. By now it seems to be impossible to obtain exact analytic expressions for $P_{\mathrm{F}}(c, t)$. The problem defies exact solution even in the long time limit $P_{\mathrm{F}}(c, \infty)$.

We also studied the distribution of the IPR:

$$
P_{\text {IPR }}(c)=\overline{\delta\left(c-\sum|\langle s \mid \mu\rangle|^{4}\right)} .
$$

Since in the long time limit the average fidelity is identical with the mean IPR one might expect the same for their respective distributions. But this is not the case. To show heuristically that the distributions of both quantities are different we look at their second moments. For the IPR it is given by $\sum_{\nu, \mu} \overline{|\langle s \mid \mu\rangle|^{4}|\langle s \mid \nu\rangle|^{4}}$ but for fidelity in the long

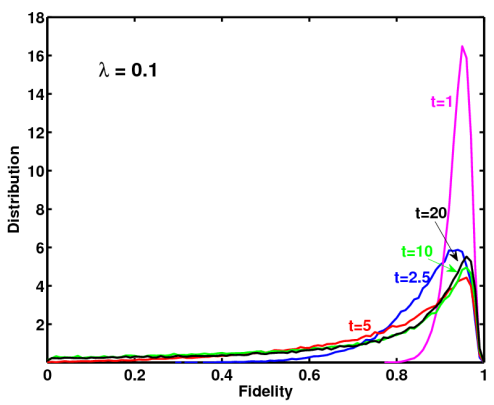

Fig. 4. Evolution in time of the fidelity distribution (25). The numerically obtained distribution function is plotted for different times $(t=1,2.5,5,10,20)$. The difference between the green curve $t=10$ and the black curve (20) is already small. Pictures taken from Ref. [33].

time limit twice this value is obtained since in the square of fidelity (21) the cross terms contribute. Therefore, although $\overline{I P R}=\overline{F(\infty)}$ (see Eq. (22)) holds, we have

$$
P_{\text {IPR }}(c) \neq P_{\mathrm{F}}(c, \infty) \text {. }
$$

For small perturbations we might approximate the distribution of the IPR as follows: as the coupling strength decreases the fragmentation of the initial state becomes smaller and smaller. The IPR will be dominated by less and less terms and finally for $\lambda \ll 1$ by only one term. This term will be the overlap of the initial state $|s\rangle$ with itself evolved adiabatically with $\lambda$. We call it $|\sigma\rangle$, see Fig. 5.

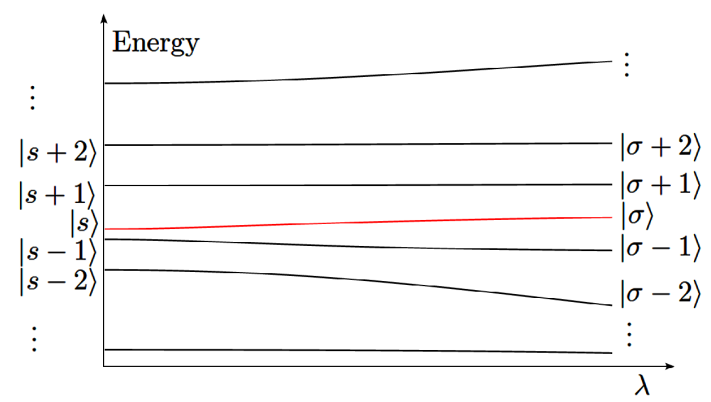

Fig. 5. Sketch of the motion of the eigenvalues and eigenvectors as a function of the dimensionless parameter $\lambda$. The initial state $|s\rangle$ evolves adiabatically to $|\sigma\rangle$.

The IPR will therefore be better and better approximated by the fourth power of the overlap $|\langle s \mid \sigma\rangle|$. Likewise, in this limit the IPR-distribution should be well approximated by the distribution of the overlap of the initial state with itself, defined as

$$
P_{c_{0}^{4}}(c)=\overline{\delta\left(c-|\langle s \mid \sigma\rangle|^{4}\right)} .
$$

For the distribution $P_{c_{0}^{4}}(c, t)$ analytical formulae could be obtained for a Poissonian background as well as for a GOE and for a GUE background [32]. For instance for a Poissonian background and for real Gaussian distributed 
coupling matrix elements $V_{i}$ the distribution is given by

$$
P_{c_{0}^{4}}(c)=\sqrt{\frac{2}{\pi}} \frac{\lambda}{2} \sqrt{\frac{\sqrt{c}}{(1-\sqrt{c})^{3}}} \exp \left(-(2 \lambda)^{2} \frac{\sqrt{c}}{1-\sqrt{c}}\right) .
$$

It has a typical essential singularity at $c=1$, which features as well for the other background Hamiltonians. Formula (29) is plotted in Fig. 6 together with the case of Poissonian background and complex $V_{i}$. We refer the reader to Ref. [32] for plots and the analytic expressions in the other cases.

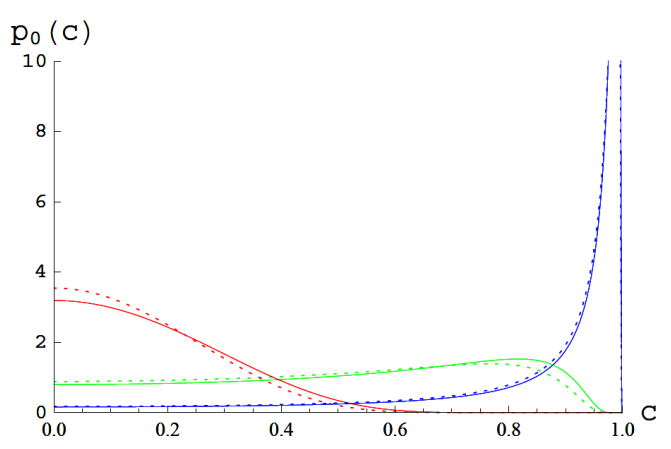

Fig. 6. Distribution function $P_{c_{0}^{4}}(c, t)$ for a regular background Hamiltonian $H_{\mathrm{b}}$ as defined in Eq. (29) for $\lambda=0.1$ (blue dotted lines), $\lambda=0.5$ (green dotted lines) and for $\lambda=1$ (red dotted lines). The full lines show the corresponding distribution for complex coupling matrix elements $V_{i}$. Pictures taken from Ref. [32].

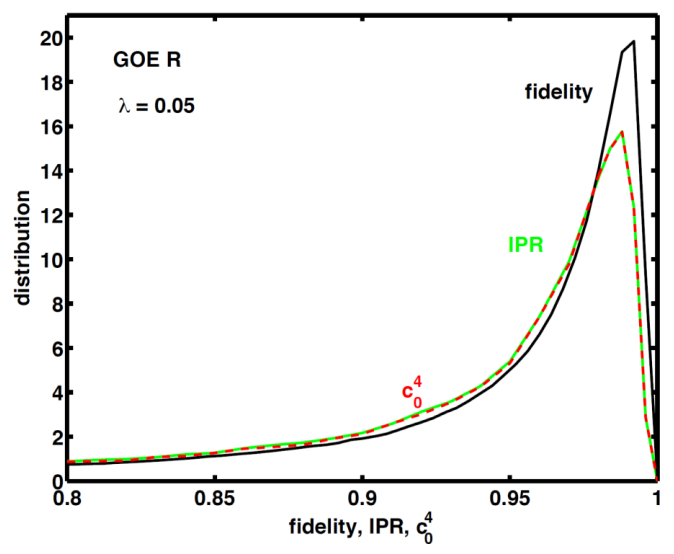

Fig. 7. Distribution functions of the fidelity in the long time limit $t \rightarrow \infty$ (full black line), of the IPR (full green line) and the distribution function $P_{c_{0}^{4}}(c, t)$ (red dashed line) defined in Eq. (28) for a GOE background and real coupling matrix elements. The mean coupling strength is very small $\lambda=0.05$. The distributions $P_{\mathrm{F}}(c, t)$ and $P_{\mathrm{IPR}}(c, t)$ were obtained numerically and $P_{c_{0}^{4}}(c, t)$ analytically. Picture taken from Ref. [33].

In Fig. 7 the three distributions $P_{\mathrm{F}}(c, \infty), P_{\mathrm{IPR}}(c)$ and $P_{c_{0}^{4}}(c)$ are plotted for small coupling strength $\lambda=0.05$. We see that $P_{\mathrm{IPR}}(c)$ and $P_{c_{0}^{4}}(c)$ coincide perfectly but
$P_{\text {IPR }}(c)$ and $P_{\mathrm{F}}(c, \infty)$, although they look similar, are substantially different.

\section{Summary and outlook}

We reviewed fidelity of a wave function in a random environment. Dependence on perturbation strength and on time differ significantly for a random initial wave and for an initial eigenfunction of $H_{0}$. In the first case fidelity amplitude could be calculated exactly. Although by now no rigorous results are available, perturbation theory seems to indicate that in this case the DrudeBoltzmann approximation is exact and fidelity is the square of fidelity amplitude [34]. As a consequence it decays to zero. This is in agreement with the general result $\overline{\mathrm{IPR}}=\overline{F(\infty)}$, since for a random initial state the IPR is zero right from the start. The question for a non-trivial distribution is related to this. If the Drude-Boltzmann approximation is exact for all powers of $f(t)$, the fidelity distribution is unavoidably a delta distribution. It is desirable to put these considerations on firm grounds.

On the other hand, fidelity of a specific prepared state saturates for $t \rightarrow \infty$, although fidelity amplitude decays to zero again. Sample to sample fluctuations are therefore important and the distribution function is a non-trivial function. It seems by now impossible to calculate the time dependent distribution function.

The distribution function of fidelity in the long time limit differs from the distribution function of the IPR. The latter approaches for small perturbation more and more the maximum overlap distribution, which is an object of high current interest $[25,35]$. To obtain analytical results for either of these distributions is hard but might be possible.

Nowadays fidelity experiments are performed by several groups. It should be possible to confirm the simple relation (10) between spectral correlations and fidelity experimentally. It might then be used to facilitate forthcoming fidelity measurements.

\section{Acknowledgments}

We acknowledge financial support by the German Research Council (DFG) with grant number KO3538/1-2.

\section{References}

[1] A. Peres, Phys. Rev. A 30, 1610 (1984).

[2] T. Gorin, T. Prosen, T.H. Seligman, M. Znidaric, Phys. Rep. 435, 33 (2006).

[3] P. Jaquod, C. Petitjean, Adv. Phys. 58, 67 (2009).

[4] H.J. Stöckmann, Quantum Chaos: An Introduction, 1st ed., Cambridge University Press, Cambridge 1999.

[5] A.M. Tulino, S. Verdú, Random Matrix Theory and Wireless Communication, now Publishers Inc, Hanover, MA 2004.

[6] O.I. Lobkis, R.L. Weaver, Phys. Rev. Lett. 90, 254302 (2003). 
[7] O. Lobkis, I. Rozhkov, R. Weaver, Phys. Rev. Lett. 91, 194101 (2003).

[8] A. Goussev, K. Richter, Phys. Rev. E 75, 015201(R) (2007).

[9] A. Goussev, D. Waltner, K. Richter, R. Jalabert, New J. Phys. 10, 093010 (2008).

[10] T. Gorin, T. Prosen, T.H. Seligman, New J. Phys. 6, 20 (2004).

[11] R. Schäfer, T. Gorin, T.H. Seligman, H.-J. Stöckmann, New J. Phys. 7, 152 (2005)

[12] T. Gorin, T.H. Seligman, R.L. Weaver, Phys. Rev. E 73, 015202(R) (2006)

[13] R.A. Jalabert, H.M. Pastawski, Phys. Rev. Lett. 86 , 2490 (2001).

[14] B.D. Simons, A. Hashimoto, M. Courtney, D. Kleppner, B.L. Altshuler, Phys. Rev. Lett. 71, 2899 (1993).

[15] M. Faas, B.D. Simons, X. Zotos, B.L. Alshuler, Phys. Rev. B 48, 5439 (1993).

[16] B.D. Simons, P.A. Lee, B.L. Altshuler, Phys. Rev. Lett. 72, 64 (1994).

[17] N. Taniguchi, A. Hashimoto, D.B. Simons, B.L. Altshuler, Europhys. Lett. 27, 335 (1994).

[18] N. Taniguchi, B.S. Shastry, B.L. Altshuler, Phys. Rev. Lett. 75, 3724 (1995).

[19] N. Taniguchi, B.D. Simons, B.L. Altshuler, Phys. Rev. B 53, R7618 (1996).

[20] H. Kohler, I.E. Smolyarenko, C. Pineda, T. Guhr, F. Leyvraz, T.H. Seligman, Phys. Rev. Lett. 100, 190404 (2008).

[21] H.J. Stöckmann, R. Schäfer, New J. Phys. 6, 199 (2004).

[22] B.D. Simons, B.L. Altshuler, in: Proc. Les-Houches Summer School, Session LXI, Eds. E.E. Akkermans, G. Montambaux, J.-L. Pichard, J. Zinn-Justin, Elsevier Science, Oxford 1995.
[23] H. Kohler, T. Nagao, H.J. Stöckmann, Phys. Rev. E 84, 061133 (2011)

[24] T. Prosen, M. Znidarič, Phys. Rev. Lett. 94, 044101 (2005).

[25] S. Åberg, T. Guhr, M. Miski-Oglu, A. Richter, Phys Rev. Lett. 100, 204101 (2008).

[26] H. Kohler, H.-J. Sommers, S. Åberg, J. Phys. A 43 , 215102 (2010).

[27] J.L. Gruver, J. Aliaga, H.A. Cerdeira, P.A. Mello, A.N. Proto, Phys. Rev. E 55, 6370 (1997)

[28] H.J. Stöckmann, R. Schäfer, Phys. Rev. Lett. 94, 244101 (2005).

[29] S. Wu, A. Tonyushkin, M.G. Prentiss, Phys. Rev. Lett. 103, 034101 (2009).

[30] A. Goussev, P. Jacquod, Phys. Rev. A 82, 053114 (2010).

[31] V.N. Prigodin, B.L. Altshuler, K.B. Efetov, S. Iida, Phys. Rev. Lett. 72, 546 (1994).

[32] H. Kohler, T. Guhr, S. Åberg, New J. Phys. 12 , 073026 (2010).

[33] H. Kohler, H.-J. Sommers, S. Åberg, T. Guhr, Phys. Rev. E 81, 050103 (2010).

[34] T. Seligman, T. Prosen, private communication.

[35] E. Bogomolny, B. Dietz, T. Friedrich, M. Miski-Oglu, A. Richter, F. Schaefer, C. Schmit, Phys. Rev. Lett. 97, 254102 (2006). 